\title{
Progesterone levels and days to luteolysis in mares treated with intrauterine fractionated coconut oil
}

\author{
Mariana Diel de Amorim ${ }^{\mathrm{a}, *, 1}$, Kayla Nielsen ${ }^{\mathrm{a}}$, Raissa Karolliny Salgueiro Cruz ${ }^{\mathrm{b}}$, \\ Claire Card ${ }^{\mathrm{a}}$ \\ ${ }^{a}$ Department of Large Animal Clinical Sciences, Western College of Veterinary Medicine, University of Saskatchewan, Saskatoon, \\ Saskatchewan, Canada \\ ${ }^{\mathrm{b}}$ Department of Veterinary Science, School of Veterinary Medicine and Animal Science, São Paulo State University (UNESP), Botucatu, \\ São Paulo, Brazil
}

\section{A R T I C L E I N F O}

\section{Article history:}

Received 14 January 2016

Accepted 3 February 2016

\section{Keywords:}

Coconut oil

Days to luteolysis

Mare

Progesterone

\begin{abstract}
A B S T R A C T
Intrauterine plant oil infusion, including fractionated coconut oil, has been previously found to be a safe, inexpensive, and reversible method of prolonging the luteal phase in mares when administered on Day 10 of the estrous cycle. Our objective was to understand the uteroovarian response to the administration of fractionated coconut oil infusion in the uterus of diestrous mares. We hypothesized that intrauterine coconut oil administration on Day 10 would prolong luteal life span in a dose-dependent fashion and would result in higher serum progesterone levels than untreated mares at the expected time of luteolysis. Light-horse mares $(n=18)$ were examined using transrectal palpation and ultrasonography to determine if they had a normal interovulatory interval and were then examined daily in estrus until the day of ovulation (Day 0 ) and then every other day during an estrous cycle. Jugular blood was drawn on Day 11, Day 13, Day 15, and Day 17, centrifuged, and serum stored until assayed for progesterone (P4; Siemens Coat-a-Count Progesterone RIA, Los Angeles, CA, USA). Mares were randomly assigned to treatment and studied over one to two estrous cycles with a rest cycle after each treatment cycle. Groups were: control $(n=5)$, fractionated coconut oil $1.0 \mathrm{~mL}$ (Miglyol 810; Sasol Oil, Witten, Germany) infused in the uterus with an artificial insemination pipette on Day 10 (Group $1 ; \mathrm{n}=5$ ) and fractionated coconut oil $0.5 \mathrm{~mL}$ infused in the uterus with an embryo transfer gun, on Day 10 (Group 2; $\mathrm{n}=5$ ). All statistical analyses were performed using analytical software (Stata SE, version 13.1, College Station, TX, USA) at $\mathrm{P}<0.05$. Data were first evaluated using the Shapiro-Wilk test for normality. Differences between groups in days to luteolysis (DTL) were examined using analysis of variance and Bonferroni, and the effect of day and treatment on P4 levels were examined using the Kruskal-Wallis and Dunn's all pairwise test. There was a significant difference in DTL between the groups $(P=0.0083)$, with fewer DTL in Group 1 compared to control $(\mathrm{P}=0.011)$ and to Group $2(\mathrm{P}=0.034)$. There was a significant effect of day ( $\mathrm{P}<0.0001)$ on P4 levels with Day $11 \mathrm{P} 4$ levels higher than Day 15 and Day 17 and Day $13 \mathrm{P} 4$ levels higher than Day 15 or Day $17(\mathrm{P}<0.03)$. There was a significant effect of treatment group $(\mathrm{P}=0.0098)$ on $\mathrm{P} 4$ levels with control and Group 2 levels higher than Group 1 mares $(\mathrm{P}=0.0012, \mathrm{P}=0.0495$, respectively). We concluded that intrauterine administration of $1 \mathrm{~mL}$ of fractionated coconut oil lowered P4 levels in diestrus in a dose-dependent fashion and did not prolonged the luteal phase of the mares.
\end{abstract}

(c) 2016 Elsevier Inc. All rights reserved.
Submitted in abstract form to the Society for Theriogenology (SFT) Annual Conference, San Antonio, TX, USA, August 2015.

* Corresponding author. Tel.: (902) 566-0458; fax: (902) 620-5053.

E-mail address: mariana.dieldeamorim@usask.ca (M. Diel de Amorim).

\footnotetext{
${ }^{1}$ Present address: Department of Health Management, Atlantic Veterinary College, University of Prince Edward Island, 550 University Avenue, Charlottetown, PE, Canada, C1A 4P3.
} 


\section{Introduction}

Estrus suppression in the mare has only been truly effective and reversible when achieved by administration of antigonadotrophin releasing hormone vaccination [1], application of treatments to prolong luteal function or by the administration of exogenous progesterone/progestagen [2]. Methods for estrus suppression through luteal lifespan lengthening in the mare consists of the use of intrauterine devices, such as marbles [2-4] or water-filled polypropylene balls [5], manual reductions of pregnancies from Days 16 to 24 after ovulation [6], oxytocin therapy in mid luteal phase [7-10] or through a prolonged 29-day oxytocin treatment [11], administration of human chorionic gonadotropin to induce a diestrous ovulation [12], and intrauterine plant oil infusion on D10 after ovulation [13].

The use of intrauterine devices has been suggested to prolong luteal function $[3,5]$ by decreasing cyclooxygenase2 (COX-2) gene expression [14]. The effectiveness of the devices has been reported to vary among studies, ranging from $11 \%$ to $41.3 \%$ [2,3]. However, several recent reports have found the deleterious effects of intrauterine glass marbles on the endometrium and future fertility of mares, where pyometra and glass fragments becoming embedded in the endometrium were described as consequences related to the prolonged use of such devices [15-19].

Manual reductions of pregnancies from Days 16 to 24 after ovulation [6] require breeding and may not be ethically acceptable for owners; hence, this method is not widely used.

Serial oxytocin injections have found to be a cheap, effective, and reversible way to prolong the luteal phase of mares when administered from Day 7 to Day 14 after ovulation once or twice a day [7-9]. The mid luteal phase oxytocin treatment has been shown to be associated with decreased plasma 13, 14-dihydro-15-keto-PGF2alpha (PGFM) concentrations and reduced endometrial COX-2 expression [20]. A recent report by Vanderwall et al. [11] (2016) reported that 29 consecutive daily treatments of oxytocin starting at any stage of the estrous cycle is as efficacious as the protocol of injecting mares with oxytocin from Day 7 to Day 14 after ovulation. Oxytocin treatment has been reported to have an efficacy of $60 \%$ to $83 \%$ in prolonging diestrus in mares [7-10]; however, these methods require multiple injections and may be problematic in mares that are adverse to intramuscular injections. Human chorionic gonadotropin administration has been used in early diestrus to induce a second ovulation [12]. The treatment is effective as long the mare has a large follicle that responds to the hormone in early diestrus, and luteal tissue of the newly formed diestrous second corpus luteum is immature and nonresponsive to the endometrial secretion of prostaglandin at Days 14 to 15 after ovulation [12].

Research performed by Wilsher and Allen in 2011, reported that the intrauterine infusion of plant oils, such as fractionated coconut oil on Day 10 after ovulation in mares was an effective and practical method of prolonging luteal function in 11 of 12 (92\%) of mares [13]. A variety of similar plant oils had similar effect on luteal function in mares when administered on Day 10 [13]. No other studies have reported the mechanism by which the intrauterine infusion of plant oils on Day 10 after ovulation prevents luteolysis and maintains endogenous levels of progesterone to suppress estrus or whether this effect is dose dependent. Therefore, the objective of the study was to compare and understand the uteroovarian response to two different amounts of fractionated coconut oil administered into the uterus of mares on Day 10 after ovulation.

\section{Materials and methods}

\subsection{Animals}

The experimental protocol was approved by the Institutional Animal Care Committee of the University of Saskatchewan. Mares $(n=26)$ of Quarter Horse, Paint, Warmblood, Thoroughbred, and Arabian breeds with a mean age of 12 years and ranging in age from 4 to 28 years were followed throughout multiple estrous cycles in 2013 and 2014. In 2013, a preliminary study was performed with eight Quarter horse mares and a total of 10 estrous cycles. Mares received $1 \mathrm{~mL}$ of sesame oil (2 cycles), coconut oil (4 cycles), or fractionated coconut oil (Mygliol 810; Sasol Oil; 4 cycles). In 2014, mares ( $\mathrm{n}=18$ ) were randomly assigned to treatment and studied over one to two estrous cycles with a rest cycle after each treatment cycle. Groups were: diestrus control $(\mathrm{n}=5)$, fractionated coconut oil $1.0 \mathrm{~mL}$ (Group 1; $\mathrm{n}=5$ ), and fractionated coconut oil $0.5 \mathrm{~mL}$ (Group 2; $\mathrm{n}=5$ ). The three remaining mares also received fractionated coconut oil (1.0 mL); however, two mares had an endometrial biopsy and biopsy cytology performed a day later, and the third mare had an endometrial biopsy and biopsy cytology performed 4 days after infusion; therefore, these mares were excluded from the data analysis and were used solely for the purpose of examining the endometrial reaction to the fractionated coconut oil.

\subsection{Reproductive procedures}

Mares were followed throughout multiple estrous cycles using transrectal palpation and ultrasonography of the reproductive tract to determine if they had a normal interovulatory interval, and were then examined daily in estrus until the day of ovulation (Day 0), and then every other day during an estrous cycle. Mares in the control group only had transrectal ultrasound examinations of the reproductive tract and blood collections performed. On Day 10 after ovulation, mares in Groups 1 and 2 were followed using transrectal palpation and ultrasonography of the reproductive tract, the perineum was aseptically prepared using multiple washes with gentle soap (Jergens, Cincinnati, $\mathrm{OH}$, USA), rinsed with water, and dried with paper towels. Mares in Group 1 had $1 \mathrm{~mL}$ of fractionated coconut oil (Miglyol 810, Sasol Oil) infused into the uterine body. Intrauterine infusion was performed with a sterile artificial insemination pipette (Minitube International, Ingersoll, ON, Canada) aseptically in the same fashion as an embryo transfer procedure. One milliliter $(1 \mathrm{~mL})$ of fractionated coconut oil was withdrawn with a sterile syringe and $18 \mathrm{G}$ needle from a sterile bottle and placed into a sterile conical tube (Corning Centrifuge Tubes; 132 Corning Incorporated Life Science, Tewksbury, MA, USA). The tip of the sterile 
artificial insemination pipette was placed into the tube and using a sterile syringe attached to the other end of the pipette, $1 \mathrm{~mL}$ of fractionated coconut oil was aspirated. The intrauterine infusion of the oil was performed in a doubleguarded fashion using an outer open-ended rectal sleeve to shield the pipette as it was advanced through the vagina near the cervix. The outer sleeve was retracted to expose an inner rectal sleeve and the insemination pipette held guarded in a hand covered by a sterile surgical glove, and the pipette was then introduced through the cervix into the uterus being careful to minimally manipulate the cervix. Mares in Group 2 had $0.5 \mathrm{~mL}$ of fractionated coconut oil (Miglyol 810, Sasol Oil) loaded into a sterile $0.5 \mathrm{~mL}$ insemination straw which was infused into the uterine body using an embryo transfer gun (IMV Technologies, Maple Grove, MN, USA) as previously described for the mares in Group 1, in the same fashion as an embryo transfer procedure. After oil infusion, all mares had a transrectal massage performed for 30 seconds to help distribute the oil within the endometrial lumen, followed by a transrectal ultrasound examination to confirm the presence and distribution of the oil in the uterine lumen (Fig. 1A-C).

A transovarian luteal biopsy (TVLB) was performed on Day 12 and Day 15 in mares in 2014, including the control group as previously described [21]. The TVLB samples collected in this study were obtained to examine the effects of the fractionated coconut oil on gene expression in luteal tissue for another study.

\subsubsection{Laboratory procedures}

2.2.1.1. Progesterone analysis. In preliminary study performed in 2013, no hormone analysis was performed. In 2014, jugular blood was collected from all mares on Day 11, Day 13, Day 15, and Day 17 after ovulation. Blood was centrifuged for 10 minutes after clotting and serum was stored at $-20{ }^{\circ} \mathrm{C}$ until analysis for progesterone. The progesterone analysis was performed at the endocrinology laboratory at the Western College of Veterinary Medicine in a single radioimmunoassay (Siemens Coat-a-Count Progesterone RIA, Los Angeles, CA, USA).

2.2.1.2. Endometrial biopsy. Endometrial biopsies were performed in three mares. Two mares had an endometrial biopsy, and biopsy cytology performed 1 day after oil infusion. One mare had an endometrial biopsy, and biopsy cytology performed 4 days after oil infusion. All endometrial biopsies were fixed in $10 \%$ buffered formalin and processed routinely for hematoxylin and eosin by Prairie Diagnostic Services Inc., Saskatoon, SK, Canada and for oil red $\mathrm{O}$ by the Animal Health Diagnostic Center, Cornell University.

\subsection{Statistics}

All statistical analyses were performed using analytical software (Stata SE, version 13.1, College Station, TX, USA) at $\mathrm{P}<0.05$. Data were evaluated using the Shapiro-Wilk test for normality. Days to luteolysis (DTL) was examined using analysis of variance, and post hoc analysis was performed using Bonferroni. Luteolysis was defined as a progesterone value below $2.0 \mathrm{ng} / \mathrm{mL}$. The effect of group and day on P4 levels were examined using the Kruskal-Wallis tests and post hoc Dunn's all pairwise test.

\section{Results}

The DTL are shown in Table 1 and were (mean \pm SD) Control (15.8 \pm 1.09 ), Group 1 (1.0 mL fractionated coconut oil; $12.2 \pm 0.45)$, and Group 2 (0.5 $\mathrm{mL}$ fractionated coconut oil; $15.2 \pm 2.48$ ). There was a significant difference in DTL between the groups ( $P=0.0083$ ), with a shorter number of days in Group 1 compared to control $(P=0.011)$, and when compared to Group $2(\mathrm{P}=0.034)$.

Table 1 shows the overall median P4 level (median [quartiles] ng/mL) from Days 11, 13, 15, and 17 in control-, Group 1-, and Group 2-treated mares. There was a significant effect of group $(P=0.0098)$ and day $(P<0.0001)$ on P4. Post hoc analysis reported that both control and Group 2 mares had higher P4 levels than Group 1 mares ( $P=0.0012, P=0.0495$, respectively). Regarding day effects, the Day 11 P4 levels were higher than Day 15, and Day 17 and Day 13 P4 levels were higher than Day 15 or Day 17 $(P<0.03)$. The oil of all types was easily visualized after infusion as a hyperechoic fluid in the uterine lumen by transrectal ultrasound (Fig. $1 \mathrm{~A}-\mathrm{C}$ ). The oil was often visible for a number of days after infusion (Fig. 1C).
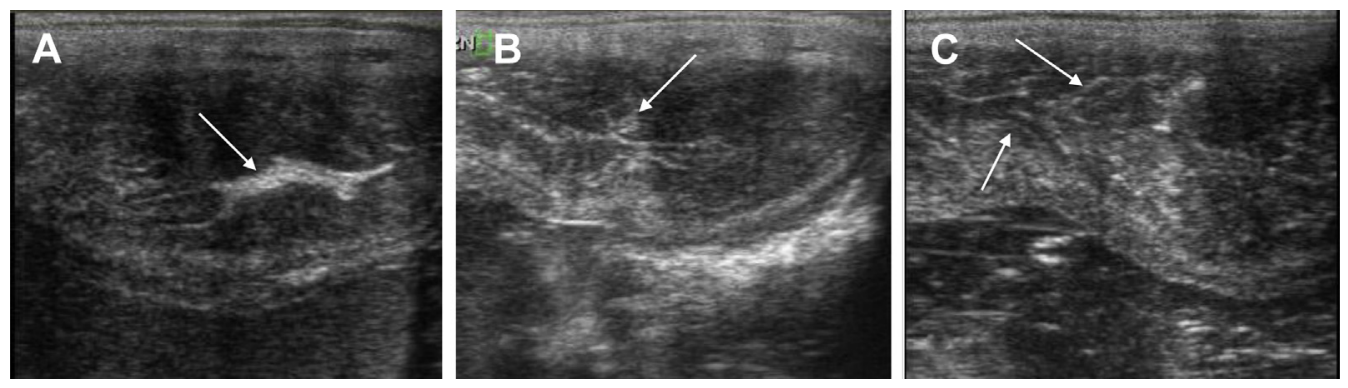

Fig. 1. (A) Transverse ultrasound image of the uterine body of a mare immediately after oil infusion showing the bright echogenic appearance of the oil (arrow) in the uterine lumen; (B) transverse ultrasound image of the uterine body of a mare after oil infusion after transrectal uterine massage showing the distribution of the oil (arrow) into the endometrial folds; (C) transverse ultrasound image of the uterine body of a mare 48 hours after oil infusion showing the echogenic oil (arrow) dispersed in the endometrial folds. 


\section{Table 1}

Number of mare cycles in control, Group 1 intrauterine infusion of $1.0 \mathrm{~mL}$ fractionated coconut oil, and Group 2 intrauterine infusion with $0.5 \mathrm{~mL}$ fractionated coconut oil on Day 10 and the subsequent days to luteolysis (DTL; mean + SD), and median (quartiles) levels of progesterone (P4) from Days $11,13,15$, and 17 after ovulation.

\begin{tabular}{llll}
\hline Group & $\begin{array}{l}\text { Number of } \\
\text { mare cycles }\end{array}$ & $\begin{array}{l}\text { DTL } \\
(\text { mean }+ \text { SD) }\end{array}$ & $\begin{array}{l}\text { P4 level } \\
\text { (median [quartiles]; ng/mL) }\end{array}$ \\
\hline Control & 5 & $15.8+1.009^{\mathrm{a}}$ & $8.0(1.5,13.8)^{\mathrm{a}}$ \\
Group 1 & 5 & $12.2+0.45^{\mathrm{b}}$ & $0.7(0.005,6.1)^{\mathrm{b}}$ \\
Group 2 & 5 & $15.2+2.48^{\mathrm{a}}$ & $3.9(0.35,12.3)^{\mathrm{a}}$ \\
\hline
\end{tabular}

${ }^{\mathrm{a}, \mathrm{b}}$ Statistically different.

An evaluation of the endometrial biopsies for inflammatory changes reported chronic mild lymphoplasmacytic inflammatory cell infiltrates and mild-to-moderate neutrophilic inflammation in the stratum compactum at 24 and 96 hours after treatment, respectively. Occasional eosinophils were present in the stratum compactum. The fractionated coconut oil could not be identified within endometrial cells in the histological specimens stained using hematoxylin and eosin or oil red $\mathrm{O}$ (Fig. 2A-D). There were either no visible or a few degenerate neutrophils present in the endometrial biopsy cytologies (Fig. 2E-F).

\section{Discussion}

Estrus suppression is desirable for: performance mares, inhibiting undesirable behavior that some mares may display in heat, or the prevention of breeding [22]. Prolongation of luteal function may occur spontaneously in mares [23]. Several options have been investigated, to reliably induce prolongation of the CL [2]. The use of intrauterine infusion of plant oils was reported to be an inexpensive, reversible means of prolonging luteal function [13]. However, we failed to replicate the results of these investigators, and in addition, there were no mare cycles $(n=25)$ in either year of this study where a mare exhibited a prolonged luteal phase. Our findings found that progesterone levels decreased at the expected time of luteolysis in those mares treated with fractionated coconut oil, with an early return to estrus in mares treated with the $1 \mathrm{~mL}$ dose of the fractionated coconut oil. The results from the preliminary study, performed in 2013, comparing different oils (sesame, coconut and fractionated coconut oil) infused into the uterus at Day 10 after ovulation in eight mare cycles yielded the same results. The fractionated coconut oil used in this study had the same chemical and fatty acid composition as published by Wilsher et al. [13] and was the identical product (Miglyol 810, Sasol Oil). Due to the fact that a majority of the mares that had the $1 \mathrm{~mL}$ intrauterine fractionated coconut oil infusion had a shorter DTL, and to rule out that the fractionated oil was not a source of bacteria, the fractionated oil was submitted to Prairie Diagnostic Services Inc. for culture and sensitivity and yielded no microbial growth. In addition, these mares were selected for good reproductive health, and none of the mares in previous cycles before oil administration were noted to have intrauterine fluid accumulations or an early return to estrus.

The intrauterine oil infusion technique used was the same as a nonsurgical embryo transfer, which has been used successfully over many years by the investigators to establish pregnancies, where aseptic preparation of mare's perineum, a double-guarded introduction of the pipette into the uterine body, and minimal dilation of the cervix were part of the routine. Our practice performs commercial embryo transfer using the same technique successfully; and therefore, we are convinced that the technique for the intrauterine oil infusion was not a contributing factor for the failure to prolong the luteal phase. The use of the embryo transfer gun to deliver the fractionated oil in Group 2 was to allow a precise delivery of a small amount of fractionated oil, which we believed would be difficult to achieve using the pipette method. When an embryo transfer gun with a $0.5 \mathrm{~mL}$ straw was used to infuse the fractionated coconut oil in the mares in Group 2, these mares also failed to have prolonged luteal phases and had no difference on either progesterone levels or DTL compared to the control animals. These data suggest either the lower dose of the fractionated coconut oil or the different infusion equipment or the combination resulted in that response. The investigators believe that the effect of the fractionated coconut oil when delivered on Day 10 of diestrus is probably dose dependent, with higher volumes being more likely to induce an early return to estrus, and that lower volumes of $0.5 \mathrm{~mL}$ or less have no effect on the DTL. Although the number of mare biopsies studied is small, the mild neutrophilic reaction noted in the mare's endometrial biopsies after oil infusion indicated that an inflammatory response occurred as a result of treatment. The endometrial inflammatory response to the fractionated coconut oil may be the underlying cause of the shorter number of DTL in the mares treated with $1 \mathrm{~mL}$ of the fractionated oil. More mare cycles would need to be studied to confirm these results.

The TVLB performed in some of the mares did not contribute to early luteal regression, as we have published recently in a larger study where both diestrus and pregnant mares underwent identical procedures, on the same days, without signs of early luteal regression or pregnancy loss [21]. In addition, none of the control mares in this report which also had TVLB had premature luteolysis. Group 1 mares had shorter DTL and lower levels of P4 than control mares, demonstrating that the fractionated coconut oil actually did not prolong the luteal phase, but it decreased it significantly when $1.0 \mathrm{~mL}$ of oil was delivered in this fashion.

The mechanism by which the fractionated coconut oil would act on the endometrium to suppress or induce $\mathrm{PGF}_{2-\alpha}$ release is unknown. It was hypothesized by Wilsher et al. [13] in 2011 that the oil would coat the luminal epithelium of the endometrium and physically interfere with the production or release of prostaglandin from the glandular epithelium, or that the various plant oils which contain poly unsaturated fatty acids (PUFA), could have an inhibitory action on COX-1 and COX-2. In human myometrium in vitro exposure to long-chain polyunsaturated acids (n-3 PUFA) such as decosohexaneoic acid (DHA) and arachidonic acid reduces prostaglandin E2 and $\mathrm{PGF}_{2-\alpha}$ significantly [24]. Penrod et al. [25] (2013) reported on the effects of the fatty acids added to equine endometrial explant cultures that were then challenged with oxytocin. The fatty acid DHA lowered PGF and prostaglandin E production by equine endometrial explants; however, this occurred without 


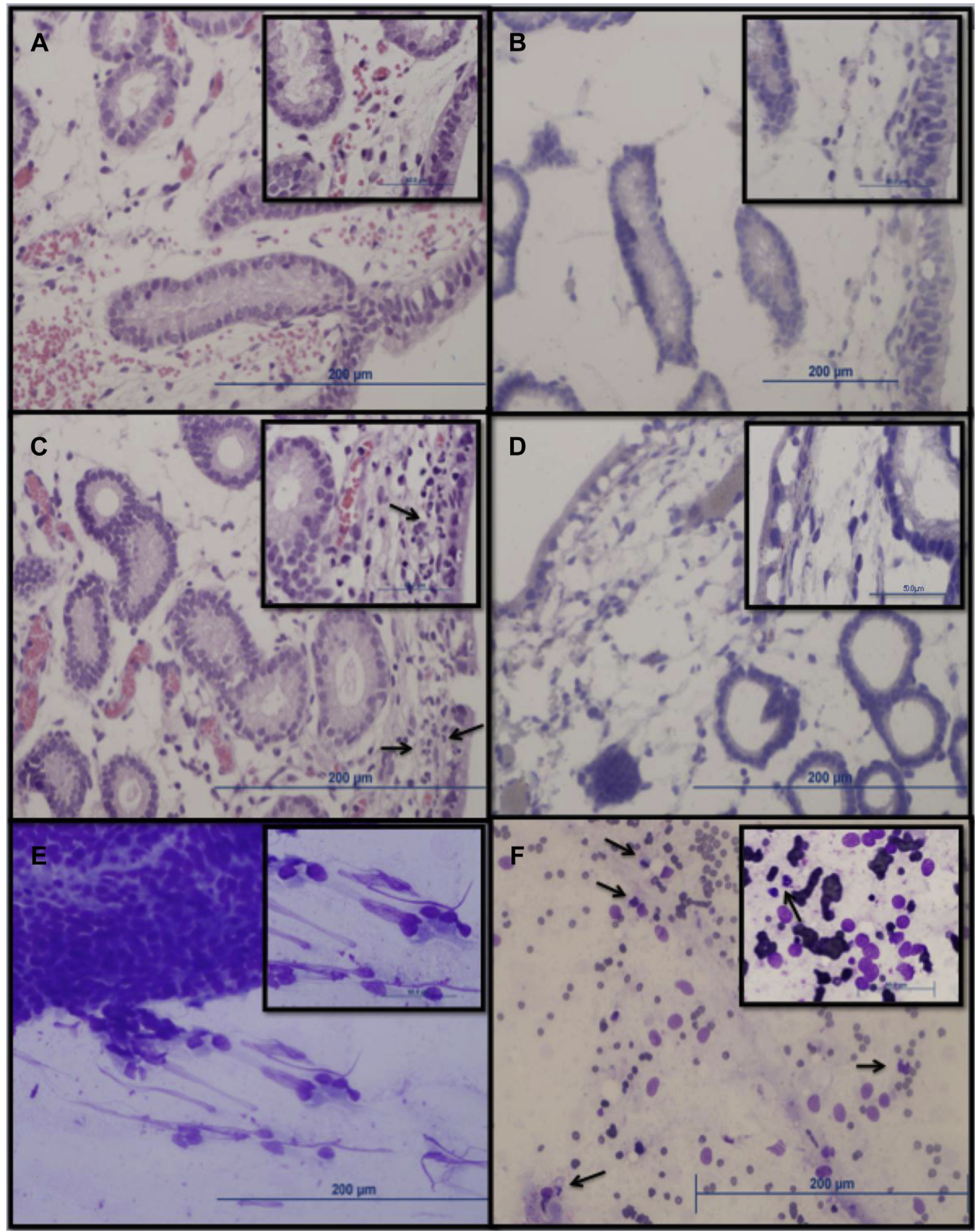

Fig. 2. (A) Hematoxylin and eosin or (B) oil red 0 stained endometrial biopsy of a mare 24 hours after fractionated coconut oil infusion; (C) hematoxylin and eosin or (D) or oil red 0 stained endometrial biopsy of a mare 96 hours after fractionated coconut oil infusion. (E) Wright Giemsa stained endometrial cytology from an impression smear from a biopsy of a mare 96 hours after fractionated coconut oil demonstrating no inflammation and from another mare (F) demonstrating mild inflammation. Arrows indicate the presence of neutrophils. 
decreasing the enzyme expression [25]. The investigators proposed that this inhibition might be due to: shifting production of PGs to a one- or three- series PG, competition of the DHA with arachidonic acid for lipid metabolism and incorporation into the phospholipid bilayer, and the incorporation of the DHA into the phospholipid bilayer influencing signaling pathways [25]. It was suggested that the plant oils of different sources with variable PUFA compositions would have different uptake rates by endometrial cells and exert different effects on prostaglandin pathways [13]. Our histologic observations of the endometrial tissues collected after treatment were that there were no visible oil containing vacuoles in the secretory endometrial epithelial cells of mares at 24 hours and 4 days after administration (Fig. 2A-D). Oil Red O staining of the endometrial biopsies also failed to demonstrate any residual oil staining material in the luminal or glandular epithelial cells, which suggested using this method that the fractionated coconut oil was either not taken up by the endometrial cells or was not detectable using this method (Fig. 2B,D). The fractionated coconut oil may have been ejected through the cervix, removed through phagocytosis, cleared through the lymphatic and circulatory system, or a combination of these mechanisms.

\subsection{Conclusion}

We concluded that intrauterine fractionated coconut oil administration on D10 of diestrus lowered progesterone levels at the $1.0 \mathrm{~mL}$ dose and shortened rather than prolonged the luteal phase of the mares.

\section{Acknowledgments}

Funding for this paper was from the Equine Health Research Fund (grant number: 414568) at the University of Saskatchewan, Western College of Veterinary Medicine. We would like to thank to Susan Cook for performing the progesterone analysis.

\section{Competing Interests}

The authors declare that they have no conflict of interest that could perceive as prejudicing the impartiality of the research reported.

\section{References}

[1] Annandale $\mathrm{CH}$, Guthrie AJ, Bertschinger HJ. Reversibility of the effects of GnRH vaccine used to suppress reproductive function in mares. Equine Vet J 2013;45:111-3.

[2] Nie GJ, Johnson KE, Branden TD, Wenzel GW. Use of a glass ball to suppress behavioural estrus in mares, in Proceedings. Proc Annu Convention Am Assoc Equine Pract 2001;41:246-8.
[3] Nie GJ, Johnson KE, Branden TD, Wenzel JGW. Use of an intrauterine glass ball protocol to extend luteal function in mares. J Equine Vet Sci 2003;23:266-73.

[4] Argo CM, Turnbull EB. The effect of intra-uterine devices on the reproductive physiology and behaviour of pony mares. Vet J 2010; 186:39-46.

[5] Rivera del Alamo MM, Reilas T, Kindahl H, Katila T. Mechanisms behind intrauterine device-induced luteal persistence in mares. Anim Reprod Sci 2008;107:94-106.

[6] Lefranc AC, Allen WR. Nonpharmacological suppression of oestrus in the mare. Equine Vet J 2004;36:183-5.

[7] Stout TAE, Lamming GE, Allen WR. Oxytocin administration prolongs luteal function in cycling mares. J Reprod Fertil 1999;116: 315-20.

[8] Vanderwall DK, Rasmussen DM, Woods GL. Effect of repeated administration of oxytocin during diestrus on duration of function of corpora lutea in mares. J Am Vet Med Assoc 2007;231:1864-7.

[9] Gee EK, Gillespie L, Bolwell CF. Effect of oxytocin on suppression of oestrus in mares exhibiting normal oestrous cycles. N Z Vet J 2012; 60:189-93.

[10] Vanderwall DK, Rasmussen DM, Carnahan KG. Effect of administrations of oxytocin during diestrus on corpus luteum function and endometrial oxytocin receptor concentration in cycling mares. J Equine Vet Sci 2012;32:536-41.

[11] Vanderwall DK, Parkison KC, Rigas J. How to use oxytocin treatment to prolong corpus luteum function for supressing estrus in mares. J Equine Vet Sci 2016;36:1-4.

[12] Hedberg Y, Dalin A-M, Santesson M, Kindahl H. A preliminary study on the induction of dioestrous ovulation in the mare - a possible method for inducing prolonged luteal phase. Acta Vet Scand 2006; 48:12.

[13] Wilsher S, Allen WR. Intrauterine administration of plant oils inhibits luteolysis in the mare. Equine Vet J 2011;43:99-105.

[14] Rivera de Alamo MM, Reilas T, Kindahl H, Rodríguez-Gil JE, Rigau T, Katila T. The luteostatic effect of intrauterine devices in mares is associated with endometrial expression of COX-2. Anim Reprod Sci 2010;121S:s227-8.

[15] Klabnik-Bradford J, Ferrer MS, Blevins C, Beard L. Marble-induced pyometra in an Appaloosa mare. Clin Theriogenology 2013;5:410.

[16] Vanderwall DK. Prolonging function of the corpus luteum to suppress estrus in mares. Proc Annu Conv Am Assoc Equine Pract 2013; 59:342-9.

[17] Diel de Amorim M, Chenier T, Nairn D, Green J, Manning S, Card C, Complications related to the use of intrauterine glass marbles in mares. Proc Annu Conv Am Assoc Equine Pract 2014;60:317.

[18] Turner RM, Vanderwall DK, Stawicki R. Complications associated with the presence of two intrauterine glass balls used for estrus suppression in a mare. Equine Vet Educ 2015;27:340-3.

[19] Freeman C, Lyle S. Chronic intermittent colic in a mare attributed to uterine marbles. Equine Vet Educ 2015;27:469-73.

[20] Keith L, Ball BA, Scoggin K, Esteller-Vico A, Woodward EM, Troedsson MHT, et al. Diestrus administration of oxytocin prolongs luteal maintenance and reduces plasma PGFM concentrations and endometrial COX-2 expression in mares. Theriogenology 2013;79: $616-24$.

[21] Diel de Amorim M, Nairn D, Manning S, Dedden I, Ripley E, Nielsen K. Effects of transvaginal luteal and ovarian biopsy on health and fertility in mares. Theriogenology 2016:1030-6.

[22] Jorgensen JS, Vivrette S, Correa M, Mansmann RA. Significance of the estrous cycle on athletic performance in mares. Proc Annu Conv Am Assoc Equine Pract 1996;42:98-100.

[23] Ginther OJ. Prolonged luteal function in mares - a semantic quagmire. Equine Vet J 1990;22:194-7.

[24] Kim PY, Zhong M, Kim YS, Sanborn BM, Allen KGD. Long chain polyunsaturated fatty acids alter oxytocin signaling and receptor density in culture pregnant human myometrial smooth muscle cells. PLoS One 2012;7:1-8.

[25] Penrod LV, Allen RE, Turner JL, Limesand SW, Arns MJ. Effects of oxytocin, lipopolysaccharide (LPS), and polyunsaturated fatty acids on prostaglandin secretion and gene expression in equine endometrial explant cultures. Domest Anim Endocrinol 2013;44:46-55. 